\title{
Some refinements of operator inequalities
}

\section{Xiaohui Fu*, Junjian Yang}

School of Mathematics and Statistics, Hainan Normal University, Haikou, 571158, China

*Corresponding author, e-mail: 51908200@qq.com

Received 26 Sep 2016

Accepted 27 Jun 2017

ABSTRACT: In this note, we refine some operator inequalities for positive unital linear maps and then give the $p>1$ power inequality of the Ando-Li-Mathias geometric mean.

KEYWORDS: positive linear maps, arithmetic mean, geometric mean

MSC2010: 47A63 47A30

\section{INTRODUCTION}

Let $M, m$ be scalars and $I$ be the identity operator. Let $\mathscr{B}(\mathscr{H})$ denote the $C^{*}$-algebra of all bounded linear operators on a Hilbert space $(\mathscr{H},\langle\cdot, \cdot\rangle)$. Throughout this paper, a capital letter means an operator in $\mathscr{B}(\mathscr{H})$. An operator $A$ is called positive if $\langle A x, x\rangle \geqslant 0$ for all $x \in \mathscr{H}$, and we write $A \geqslant 0$. An operator $A$ is said to be strictly positive if $A>0$. For self-adjoint operators $A, B \in \mathscr{B}(\mathscr{H})$, we say $B \geqslant A$ $(B \leqslant A)$ if $B-A \geqslant 0(B-A \leqslant 0)$. The operator norm is denoted by $\|\cdot\|$. We identify the absolute value operator of $A$ with $|A|=\left(A^{*} A\right)^{1 / 2}$, where $A^{*}$ stands for the adjoint of $A$. A linear map $\Phi$ is positive if $\Phi(A) \geqslant 0$ whenever $A \geqslant 0$. It is said to be unital if $\Phi(I)=I$.

For $A, B>0$, the geometric mean $A \sharp B{ }^{1,2}$ is defined by

$$
A \sharp B=A^{1 / 2}\left(A^{-1 / 2} B A^{-1 / 2}\right)^{1 / 2} A^{1 / 2} .
$$

One motivation for such a notion is of course the operator AM-GM inequality:

$$
\frac{A+B}{2} \geqslant A \sharp B \text {. }
$$

We denote the Ando-Li-Mathias geometric mean $^{3,4}$ for $A_{1}, \ldots, A_{n}>0$ by $G\left(A_{1}, \ldots, A_{n}\right)$. There is no explicit formula for $G\left(A_{1}, \ldots, A_{n}\right)$ in terms of $A_{1}, \ldots, A_{n}$ when $n \geqslant 3$. However, the only two basic properties that we need are

$$
\begin{gathered}
G\left(A_{1}^{-1}, \ldots, A_{n}^{-1}\right)=G^{-1}\left(A_{1}, \ldots, A_{n}\right), \\
G\left(A_{1}, \ldots, A_{n}\right) \leqslant \frac{A_{1}+\cdots+A_{n}}{n} .
\end{gathered}
$$

It is well known that for two positive operators $A$ and $B$,

$$
A \geqslant B \nRightarrow A^{p} \geqslant B^{p} \quad \text { for } \quad p>1 .
$$

Let $0<m I \leqslant A \leqslant M I$ and $\Phi$ be positive unital linear map. Lin (Theorem 2.10 in Ref. 5) proved the operator inequalities

$$
\begin{aligned}
\left|\Phi\left(A^{-1}\right) \Phi(A)+\Phi(A) \Phi\left(A^{-1}\right)\right| & \leqslant \frac{1}{2} \mu, \\
\Phi\left(A^{-1}\right) \Phi(A)+\Phi(A) \Phi\left(A^{-1}\right) & \leqslant \frac{1}{2} \mu .
\end{aligned}
$$

where $\mu=(M+m)^{2} / M m$.

$\mathrm{Fu}$ (Theorem 4 in Ref. 6) presented the following generalizations of (3) and (4). Let $0<m I \leqslant A \leqslant$ $M I$ and $p \geqslant 1$. Then for positive unital linear map $\Phi$,

$$
\begin{aligned}
\left|\Psi_{p}(A)\right| & \leqslant \frac{1}{2} \mu^{p}, \\
\Psi_{p}(A) & \leqslant \frac{1}{2} \mu^{p} .
\end{aligned}
$$

where $\Psi_{p}(A)=\Phi^{p}\left(A^{-1}\right) \Phi^{p}(A)+\Phi^{p}(A) \Phi^{p}\left(A^{-1}\right)$.

Let $0<m I \leqslant A_{i} \leqslant M I$ for $i=1, \ldots, n$. Fujii ${ }^{7}$ proved the reverse operator AM-GM inequality

$$
\frac{A_{1}+\cdots+A_{n}}{n} \leqslant \frac{1}{4} \mu G\left(A_{1}, \ldots, A_{n}\right) .
$$

Lin (Theorem 3.2 in Ref. 8) revealed that the reverse AM-GM inequality (7) can be squared:

$$
\left(\frac{A_{1}+\cdots+A_{n}}{n}\right)^{2} \leqslant\left(\frac{1}{4} \mu\right)^{2} G^{2}\left(A_{1}, \ldots, A_{n}\right) .
$$

$\mathrm{Fu}$ (Theorem 5 in Ref. 6) also generalized (8) as follows. Let $0<m I \leqslant A_{i} \leqslant M I,(i=1, \ldots, n)$ and $p \geqslant 1$. Then for positive unital linear map $\Phi$,

$$
\left(\frac{A_{1}+\cdots+A_{n}}{n}\right)^{2 p} \leqslant\left(\frac{1}{4} \mu^{p}\right)^{2} G^{2 p}\left(A_{1}, \ldots, A_{n}\right) .
$$

In this paper, we will give some operator inequalities which are refinements of (5), (6) and (9). 


\section{MAIN RESULTS}

We give some Lemmas before we present the main theorems of this paper. The following two lemmas can be found in Ref. 9 (Theorem 1.6.9 and p. 39, Kadison's inequality).

Lemma 1 Let $A$ and $B$ be positive operators. Then for $1 \leqslant r<\infty$

$$
\left\|A^{r}+B^{r}\right\| \leqslant\left\|(A+B)^{r}\right\| .
$$

Lemma 2 Let $\Phi$ be positive unital linear map. Then for every self-adjoint operator $A$

$$
\Phi^{2}(A) \leqslant \Phi\left(A^{2}\right) .
$$

Furthermore, in Lemma 2 if $A$ is positive and $1 \leqslant r \leqslant$ 2 , then

$$
\Phi^{r}(A) \leqslant \Phi\left(A^{r}\right) .
$$

The next two lemmas are presented in Refs. 5, 10, 11 (Lemma 2.9 in Ref. 5).

Lemma 3 Let $A, B>0$. Then the following norm inequality holds:

$$
\|A B\| \leqslant \frac{1}{4}\|A+B\|^{2} .
$$

Lemma 4 For any bounded linear operator $X$

$$
|X| \leqslant t I \Leftrightarrow\|X\| \leqslant t \Leftrightarrow\left[\begin{array}{cc}
t I & X \\
X^{*} & t I
\end{array}\right] \geqslant 0 .
$$

Now we present the first main result in the following theorem.

Theorem 1 Let $A \geqslant 0$. Then for positive unital linear map $\Phi$,

$$
\left|\Psi_{p}(A)\right| \leqslant \begin{cases}\frac{1}{2} \mu_{p}, & 1 \leqslant p \leqslant 2, \\ \frac{1}{2} \mu_{2,1}^{p}, & p \geqslant 2,\end{cases}
$$

where $\mu_{a}=\left(M^{a}+m^{a}\right)^{2} /(M m)^{a}, \quad \mu_{a, b}=\left(M^{a}+\right.$ $\left.m^{a}\right) /(M m)^{b}$ and

$$
\Psi_{p}(A) \leqslant \begin{cases}\frac{1}{2} \mu_{p}, & 1 \leqslant p \leqslant 2, \\ \frac{1}{2} \mu_{2,1}^{p}, & p \geqslant 2 .\end{cases}
$$

Proof: We first consider the case of $p \geqslant 2$. Compute

$$
\begin{aligned}
& \left\|\Phi^{p}(A) M^{p} m^{p} \Phi^{p}\left(A^{-1}\right)\right\| \\
& \quad \leqslant \frac{1}{4}\left\|\Phi^{p}(A)+M^{p} m^{p} \Phi^{p}\left(A^{-1}\right)\right\|^{2} \quad(\text { by (13)) } \\
& \quad=\frac{1}{4}\left\|\left(\Phi^{2}(A)\right)^{p / 2}+\left(M^{2} m^{2} \Phi^{2}\left(A^{-1}\right)\right)^{p / 2}\right\|^{2} \\
& \quad \leqslant \frac{1}{4}\left\|\Phi^{2}(A)+M^{2} m^{2} \Phi^{2}\left(A^{-1}\right)\right\|^{p} \quad(\text { by (10)) } \\
& \quad \leqslant \frac{1}{4}\left(M^{2}+m^{2}\right)^{p} \quad\left(\text { by (11) and }{ }^{8}(4.6)\right) .
\end{aligned}
$$

So

$$
\left\|\Phi^{p}(A) \Phi^{p}\left(A^{-1}\right)\right\| \leqslant \frac{1}{4} \mu_{2,1}^{p} .
$$

From (14) and (16), we obtain

$$
\left[\begin{array}{cc}
\frac{1}{4} \mu_{2,1}^{p} I & \Phi^{p}(A) \Phi^{p}\left(A^{-1}\right) \\
\Phi^{p}\left(A^{-1}\right) \Phi^{p}(A) & \frac{1}{4} \mu_{2,1}^{p} I
\end{array}\right] \geqslant 0,
$$

and

$$
\left[\begin{array}{cc}
\frac{1}{4} \mu_{2,1}^{p} I & \Phi^{p}\left(A^{-1}\right) \Phi^{P}(A) \\
\Phi^{P}(A) \Phi^{P}\left(A^{-1}\right) & \frac{1}{4} \mu_{2,1}^{p} I
\end{array}\right] \geqslant 0 .
$$

Summing these two operator matrices, the matrix

$$
\left[\begin{array}{ll}
\frac{1}{2} \mu_{2,1}^{p} I & \Psi_{p}(A) \\
\Psi_{p}(A) & \frac{1}{2} \mu_{2,1}^{p} I
\end{array}\right]
$$

is positive. From (14), we achieve the operator inequality

$$
\left|\Psi_{p}(A)\right| \leqslant \frac{1}{2} \mu_{2,1}^{p} .
$$

Secondly, we consider the case of $1 \leqslant p \leqslant 2$. Compute

$$
\begin{aligned}
& \left\|\Phi^{p}(A) M^{p} m^{p} \Phi^{p}\left(A^{-1}\right)\right\| \\
& \quad \leqslant \frac{1}{4}\left\|\Phi^{p}(A)+M^{p} m^{p} \Phi^{p}\left(A^{-1}\right)\right\|^{2} \quad \text { (by (13)) } \\
& \quad \leqslant \frac{1}{4}\left\|\Phi\left(A^{p}\right)+M^{p} m^{p} \Phi\left(A^{-p}\right)\right\|^{2} \quad \text { (by (12)) } \\
& \quad \leqslant \frac{1}{4}\left(M^{p}+m^{p}\right)^{2} .
\end{aligned}
$$

The last inequality above holds as follows. Since $0<$ $m I \leqslant A \leqslant M I$ and $0<m^{p} I \leqslant A^{p} \leqslant M^{p} I$, we have

$$
\begin{aligned}
\left(M^{p}-A^{p}\right)\left(m^{p}-A^{p}\right) A^{-p} & \leqslant 0 \\
\Leftrightarrow M^{p} m^{p} A^{-p}+A^{p} & \leqslant M^{p}+m^{p},
\end{aligned}
$$

and hence

$$
M^{p} m^{p} \Phi\left(A^{-p}\right)+\Phi\left(A^{p}\right) \leqslant M^{p}+m^{p} .
$$

So

$$
\left\|\Phi^{p}(A) \Phi^{p}\left(A^{-1}\right)\right\| \leqslant \frac{1}{4} \mu_{p} .
$$

From (14) and the above inequality, we obtain

$$
\left[\begin{array}{cc}
\frac{1}{4} \mu_{p} I & \Phi^{p}(A) \Phi^{p}\left(A^{-1}\right) \\
\Phi^{p}\left(A^{-1}\right) \Phi^{p}(A) & \frac{1}{4} \mu_{p} I
\end{array}\right] \geqslant 0,
$$

and

$$
\left[\begin{array}{cc}
\frac{1}{4} \mu_{p} I & \Phi^{P}\left(A^{-1}\right) \Phi^{p}(A) \\
\Phi^{p}(A) \Phi^{p}\left(A^{-1}\right) & \frac{1}{4} \mu_{p} I
\end{array}\right] \geqslant 0 .
$$

Summing these two operator matrices, we have

$$
\left[\begin{array}{ll}
\frac{1}{2} \mu_{p} I & \Psi_{p}(A) \\
\Psi_{p}(A) & \frac{1}{2} \mu_{p} I
\end{array}\right] \geqslant 0 .
$$


From (14) we obtain

$$
\left|\Psi_{p}(A)\right| \leqslant \frac{1}{2} \mu_{p} .
$$

As $|X| \geqslant X$ for any self-adjoint $X$, (15) follows from

(1). The desired inequalities follow.

Remark 1 For any $p \geqslant 1$ in (1),

$$
\frac{1}{2} \mu_{p} \leqslant \frac{1}{2} \mu^{p},
$$

and

$$
\frac{1}{2} \mu_{2,1}^{p} \leqslant \frac{1}{2} \mu^{p}
$$

Thus (1) is the refinement of (5). Similarly, (15) is tighter than (6).

In the next theorem, we show a refinement of the reverse operator AM-GM inequality (9).

Theorem 2 Let $0<m I \leqslant A_{i} \leqslant M I(i=1, \ldots, n)$. Then for positive unital linear map $\Phi$,

$$
\begin{aligned}
& \left(\frac{A_{1}+\cdots+A_{n}}{n}\right)^{2 p} \\
& \quad \leqslant \begin{cases}\left(\frac{1}{4} \mu_{p}\right)^{2} G^{2 p}\left(A_{1}, \ldots, A_{n}\right), & 1 \leqslant p \leqslant 2, \\
\left(\frac{1}{4} \mu_{2,1}^{p}\right)^{2} G^{2 p}\left(A_{1}, \ldots, A_{n}\right), & p \geqslant 2 .\end{cases}
\end{aligned}
$$

Proof: Firstly, consider the case of $p \geqslant 2$. Compute

$$
\begin{aligned}
& \left\|\left(\frac{A_{1}+\cdots+A_{n}}{n}\right)^{p} M^{p} m^{p} G^{-p}\left(A_{1}, \ldots, A_{n}\right)\right\| \\
& \leqslant \frac{1}{4} \|\left(\left(\frac{A_{1}+\cdots+A_{n}}{n}\right)^{2}\right)^{p / 2}+ \\
& \quad\left(M^{2} m^{2} G^{-2}\left(A_{1}, \ldots, A_{n}\right)\right)^{p / 2} \|^{2}(\text { by }(13)) \\
& \leqslant \frac{1}{4} \|\left(\frac{A_{1}+\cdots+A_{n}}{n}\right)^{2}+ \\
& \leqslant \frac{1}{4} \| \frac{A_{1}^{2}+\cdots+A_{n}^{2}}{n}+ \\
& M^{2} m^{2} G^{-2}\left(A_{1}, \ldots, A_{n}\right) \|^{p}(\text { by }(10)) \\
& \quad M^{2} m^{2} G^{-2}\left(A_{1}, \ldots, A_{n}\right) \|^{p} \\
& \quad\left(\text { by operator convexity of } A^{2}\right) \\
& \leqslant \frac{1}{4}\left(M^{2}+m^{2}\right)^{p} .
\end{aligned}
$$

The last inequality above is obtained as follows. From

$$
0<m^{2} I \leqslant A_{i} \leqslant M^{2} I
$$

and

$$
\left(M^{2}-A_{i}^{2}\right)\left(m^{2}-A_{i}^{2}\right) A_{i}^{-2} \leqslant 0,
$$

it suffices to show that, for $i=1, \ldots, n$,

$$
A_{i}^{2}+M^{2} m^{2} A_{i}^{-2} \leqslant M^{2}+m^{2}
$$

and

$$
\frac{A_{1}^{2}+\cdots+A_{n}^{2}}{n}+M^{2} m^{2} \frac{A_{1}^{-2}+\cdots+A_{n}^{-2}}{n} \leqslant M^{2}+m^{2}
$$

hold. Furthermore, by (1) and (2), we have

$$
\frac{A_{1}^{2}+\cdots+A_{n}^{2}}{n}+M^{2} m^{2} G^{-2}\left(A_{1}, \ldots, A_{n}\right) \leqslant M^{2}+m^{2} .
$$

Thus

$$
\left\|\left(\frac{A_{1}+\cdots+A_{n}}{n}\right)^{p} G^{-p}\left(A_{1}, \ldots, A_{n}\right)\right\| \leqslant \frac{1}{4} \mu_{2,1}^{p} .
$$

From Ref. 12 (p. 40), we know that the last inequality is equivalent to

$$
\left(\frac{A_{1}+\cdots+A_{n}}{n}\right)^{2 p} \leqslant\left(\frac{1}{4} \mu_{2,1}^{p}\right)^{2} G^{2 p}\left(A_{1}, \ldots, A_{n}\right) .
$$

Next, consider the case of $1 \leqslant p \leqslant 2$. Compute

$$
\begin{aligned}
& \left\|\left(\frac{A_{1}+\cdots+A_{n}}{n}\right)^{p} M^{p} m^{p} G^{-p}\left(A_{1}, \ldots, A_{n}\right)\right\| \\
& \leqslant \frac{1}{4} \|\left(\frac{A_{1}+\cdots+A_{n}}{n}\right)^{p}+ \\
& \quad M^{p} m^{p} G^{-p}\left(A_{1}, \ldots, A_{n}\right) \|^{2} \text { (by (13)) } \\
& \leqslant\left\|\frac{A_{1}^{p}+\cdots+A_{n}^{p}}{n}+M^{p} m^{p} G^{-p}\left(A_{1}, \ldots, A_{n}\right)\right\|^{2}
\end{aligned}
$$

(by operator convexity of $A^{2}$ )

$$
\leqslant \frac{1}{4}\left(M^{p}+m^{p}\right)^{2} .
$$

As with the proof of the case of $p \geqslant 2$, the last inequality is obtained. So

$$
\left\|\left(\frac{A_{1}+\cdots+A_{n}}{n}\right)^{p} G^{-p}\left(A_{1}, \ldots, A_{n}\right)\right\| \leqslant \frac{1}{4} \mu_{p},
$$

which is equivalent to

$$
\left(\frac{A_{1}+\cdots+A_{n}}{n}\right)^{2 p} \leqslant\left(\frac{1}{4} \mu_{p}\right)^{2} G^{2 p}\left(A_{1}, \ldots, A_{n}\right) .
$$

Remark 2 For any $p \geqslant 1$ in (17), the following inequalities always hold:

$$
\left(\frac{1}{4} \mu_{p}\right)^{2} \leqslant\left(\frac{1}{4} \mu^{p}\right)^{2}
$$

and

$$
\left(\frac{1}{4} \mu_{2,1}^{p}\right)^{2} \leqslant\left(\frac{1}{4} \mu^{p}\right)^{2} .
$$

Thus (17) is sharper than (9). 
Acknowledgements: This study was supported by the Key Project of Applied Mathematics of Hainan Normal University, the Natural Science Foundation of Hainan Province (No. 20161005), and the Doctoral Scientific Research Foundation of Hainan Normal University.

\section{REFERENCES}

1. Moslehian MS, Fu X-H (2016) Squaring operator Pólya-Szegö and Diaz-Metcalf type inequalities. Lin Algebra Appl 491, 73-82.

2. Khosravi M (2016) Some matrix inequalities for weighted power mean. Ann Funct Anal 7, 348-57.

3. Ando T (2016) Square inequality and strong order relation. Adv Operat Theor 1, 1-7.

4. Ando T, Li C-K, Mathias R (2004) Geometric means. Lin Algebra Appl 385, 305-34.

5. Lin M-H (2013) On an operator Kantorovich inequality for positive linear maps. $J$ Math Anal Appl 402, $127-32$.

6. Fu X-H (2015) Some generalizations of operator inequalities. $J$ Math Inequal 9, 101-5.

7. Fujii JI, et al (2007) A reverse of the weighted geometric mean due to Lawson-Lim. Lin Algebra Appl 427, 272-84.

8. Lin M-H (2013) Squaring a reverse AM-GM inequality. Stud Math 215, 187-94.

9. Bhatia R (2007) Positive Definite Matrices, Princeton Univ Press.

10. Bhatia R, Kittaneh F (2000) Notes on matrix arithmetic-geometric mean inequalities. Lin Algebra Appl 308, 203-11.

11. Zhang P-P (2015) More operator inequalities for positive linear maps. Banach J Math Anal 9, 166-72.

12. Zhan X-Z (2008) Matrix Theory, Higher Education Press, Beijing [in Chinese]. 